\title{
Determination of the minimum number of lymph nodes to examine to maximize survival in patients with esophageal carcinoma: Data from the Surveillance Epidemiology and End Results database
}

\author{
Shawn S. Groth, MD, ${ }^{\text {a }}$ Beth A. Virnig, PhD, ${ }^{\text {b,c }}$ Bryan A. Whitson, MD, ${ }^{a}$ Todd E. DeFor, MS, ${ }^{c}$ \\ Zhong-ze Li, MS, ${ }^{\mathrm{c}}$ Todd M. Tuttle, MD, ${ }^{\mathrm{a}, \mathrm{c}}$ and Michael A. Maddaus, MD ${ }^{\mathrm{a}, \mathrm{c}}$
}

\begin{abstract}
Objective: We used a population-based cancer registry to examine the association between lymph node counts and mortality to determine the minimum number of lymph nodes that should be examined as part of esophageal resection.
\end{abstract}

\begin{abstract}
Methods: Using the Surveillance Epidemiology and End Results database, we identified patients who had an esophagectomy for invasive esophageal carcinoma from 1988 through 2005 and who had a known number of lymph nodes examined pathologically. After stratifying patients $(0,1-11,12-29$, and 30 or more lymph nodes examined) based on a recursive partitioning analysis, we assessed the association between lymph nodes counts and mortality using the Kaplan-Meier method. To adjust for potential confounding covariates, we used a Cox proportional hazards regression model.
\end{abstract}

Results: Of the patients in the Surveillance Epidemiology and End Results database with esophageal cancer, 4882 met our inclusion criteria. We noted a significant difference between the lymph node groups with regards to unadjusted all-cause $(P<.0001)$ and cancer-specific mortality $(P=.004)$. After adjusting for cancer registry, patient factors, tumor characteristics, and timing of radiation therapy, we noted a significant difference between the lymph node groups with regards to all-cause and cancer-specific mortality. Compared with patients who had no lymph node evaluation, only patients who had more than 12 lymph nodes examined had a significant improvement in mortality; patients who had 30 or more lymph nodes examined had significantly lower mortality rates than the other groups.

Conclusion: To maximize all-cause and cancer-specific survival, esophageal cancer patients should have at least 30 lymph nodes examined pathologically as part of esophageal resection. (J Thorac Cardiovasc Surg 2010;139: 612-20)

Earn CME credits at

http://cme.ctsnetjournals.org

Accurate esophageal cancer staging has important prognostic and therapeutic implications, yet there is no standard of care regarding the minimum number of lymph nodes (LNs) that should be removed during esophagectomy and examined by the pathologist.

We utilized a large population-based cancer registry to (1) determine the minimum number of LNs that should be examined to maximize overall and cancer-specific survival

From the University of Minnesota Department of Surgery, ${ }^{a}$ Division of Thoracic Foregut Surgery; University of Minnesota School of Public Health, ${ }^{\mathrm{b}}$ Division of Health Policy and Management; and University of Minnesota Cancer Center, ${ }^{c}$ Minneapolis, Minn.

Disclosures: None.

Received for publication Nov 12, 2008; revisions received May 27, 2009; accepted for publication July 6, 2009; available ahead of print Aug 26, 2009.

Address for reprints: Michael A. Maddaus, MD, University of Minnesota Department of Surgery, Section of Thoracic and Foregut Surgery, MMC 207, 420 Delaware St SE, Minneapolis, MN 55455 (E-mail: madda001@umn.edu).

$0022-5223 / \$ 36.00$

Copyright $\odot 2010$ by The American Association for Thoracic Surgery doi:10.1016/j.jtcvs.2009.07.017 after esophagectomy for esophageal carcinoma and (2) ascertain if this number is the same as the minimum number of LNs needed for accurate nodal staging. We hypothesized that (1) higher total LN counts are associated with improved survival to a point, after which higher counts confer no significant incremental improvement in survival and (2) the minimum number of LNs needed to maximize survival is different than the minimum number needed for accurate cancer staging.

\section{METHODS}

The Human Subjects Committee of the University of Minnesota determined that this study was exempt from formal review by the Institutional Review Board.

\section{Data}

The Surveillance, Epidemiology, and End Results (SEER) database is a population-based cancer registry that was founded in 1973 by the National Cancer Institute (Bethesda, Md). Currently, 17 US cancer registries, selected to encompass a diverse sample (about $26 \%$ ) of the national population, participate in the SEER Program. We used the SEER database that was based on the November 2007 submission, which provides data through December 31, 2005. ${ }^{1}$ SEER registries 


$$
\begin{aligned}
& \text { Abbreviations and Acronyms } \\
& \text { AJCC }=\text { American Joint Committee on Cancer } \\
& \text { CI }=\text { confidence interval } \\
& \text { GEJ }=\text { gastroesophageal junction } \\
& \text { LN }=\text { lymph node } \\
& \text { OR }=\text { odds ratio } \\
& \text { SEER }= \text { Surveillance, Epidemiology, and End } \\
& \text { Results }
\end{aligned}
$$

began collecting LN data in 1988; therefore, this study represents data collected by the SEER registries from 1988 through 2005. Because of the negative impact of Hurricane Katrina on data collection by the Louisiana SEER registry, we excluded information obtained by that registry in 2005 .

We collected information on patient characteristics, primary tumor characteristics, and treatment regimens.

\section{Inclusion Criteria}

We selected patients from the SEER database for inclusion in our study using the following International Classification Disease for Oncology, 3rd Edition (ICD-O3) ${ }^{2}$ topography codes (in parenthesis) for each anatomic location in the esophagus: proximal esophagus (15.0 and 15.3; lower border of the cricoid cartilage to the tracheal bifurcation), midthoracic esophagus (15.1 and 15.4; tracheal bifurcation midway to the gastroesophageal junction [GEJ]), distal esophagus (15.2 and 15.5; midway to the GEJ to the GEJ), overlapping lesions (15.8) and esophageal lesions, not otherwise specified (15.9).

We only included patients over the age of 18 who had an esophagectomy for invasive esophageal carcinoma and who had a known number of LNs examined (pathologically). The following carcinoma histologic subtypes were included in our analysis (ICD-O3 morphology codes in parenthesis) and were categorized as follows: (1) adenocarcinomas: papillary carcinoma, not otherwise specified (8050-8052), basaloid carcinoma (8123), adenocarcinoma (8140-8147), adenocarcinoma in an adenomatous polyp (8210), tubular adenocarcinoma (8211), adenocarcinoma with mixed subtypes (8255), papillary adenocarcinoma (8260-8263), clear cell adenocarcinoma (8310), mucinous adenocarcinoma (8480-8481), signet cell carcinoma (8490), acinar adenocarcinoma (8550), and adenocarcinoma with other features (8570-8575); (2) squamous cell carcinomas: spindle cell carcinoma (8032), squamous cell carcinoma (8070-8077), basaloid squamous carcinoma (8083), and basosquamous cell carcinoma (8094).

\section{Exclusion Criteria}

We excluded patients with more than 1 primary tumor. Due to the potential for confounding, we also excluded those patients who were unlikely to have received aggressive cancer treatment: patients with metastatic disease and patients with a hospice, nursing home, autopsy, or death certificate as their only reporting source.

\section{Statistical Analysis}

Data were analyzed using SAS version 9.1 (SAS Institute, Cary, NC). For all statistical testing, we used a 2-sided significance level (alpha) of .05. We stratified patients into LN groups with homogenous survival using a recursive partitioning analysis based on the log-rank statistic. ${ }^{3}$ A sensitivity analysis was performed to confirm that our choice of cut points did not change our study findings. Descriptive statistics were collected. Where appropriate, results are reported as mean \pm standard deviation for normally distributed variables and median (range in parenthesis) for nonparametric variables. Between-group comparisons were made using a 2-sample $t$ test or an analysis of variance for normally distributed continuous variables, a Wilcoxon rank-sum or Kruskal-Wallis test for nonparametric continuous variables, and a chi-square test for categorical variables.

To compare unadjusted all-cause mortality (death from any cause) and cancer-specific mortality rates (death from cancer) between each LN group, we used the Kaplan-Meier method. We adjusted for the following potential confounding covariates using a Cox proportional hazards regression model: patient characteristics (age, race, and gender), geographical location (cancer registry), tumor characteristics (histology, grade, American Joint Committee on Cancer [AJCC] pathological T stage, ${ }^{4}$ and anatomic location), and timing of radiation therapy. With the exception of pathologic T stage, all of the covariates are known to a surgeon prior to esophagectomy. Because of a lack of longer-term follow-up ( $12 \%$ of our cohort), we censored our survival analyses at 5 years. After testing the proportional hazards assumption, we generated log-log survival plots to subjectively assess homogeneity of the hazard ratios over time. After adjusting alpha using the Bonferroni correction, we used a multiple comparisons test to compare hazard ratios between $\mathrm{LN}$ groups. We validated our model by taking out a $33 \%$ random sample of our cohort and repeating our survival analysis.

Total LN counts (our principal explanatory variable) are highly correlated with the number of positive LNs, which in turn are highly correlated with $\mathrm{N}$ stage. Therefore, we did not include number of positive LNs (or N stage) in our model to avoid multicollinearity (which could reduce the precision of our model). Instead, we adjusted for the potential confounding influence of LN metastasis by performing a stratified survival analysis. We also assessed for interaction between other tumor characteristics (ie, T stage, histology, and grade) and LN counts. Due to concern for interaction between LN counts and timing of radiation therapy, we also stratified our results by timing of radiation therapy. We hypothesized that neoadjuvant radiation therapy was associated with lower LN counts. 
The current AJCC staging system for esophageal cancer dichotomizes nodal status: N0 (absence of LN metastasis) and N1 (presence of LN metastasis). Therefore, to assess whether the number of LNs needed to optimize survival was consistent with the number needed to optimize accurate nodal staging, we plotted the frequency of patients with at least 1 positive LN for each LN count using locally weighted least squares smoothing. We compared to odds of finding LN metastasis in each LN group using a logistic regression model adjusted for the same covariates as the Cox proportional hazards regression model.

\section{RESULTS \\ Descriptive Statistics}

Of 7323 patients in the SEER database (1988-2005) who had an esophagectomy for invasive esophageal cancer and had a known number of LNs examined, 4882 met our inclusion criteria (Figure 1). Patients who had an esophagectomy and had a known number of LNs examined that we excluded from our study were more likely to be older, to have an unknown $\mathrm{T}$ stage, an undifferentiated or unknown grade, and more positive LNs. They also had fewer LNs examined and were less likely to undergo radiation therapy (Table 1). Patients who were included in our study did not have significantly different all-cause (log-rank, $P=.43$ ) or cancer-specific (log-rank, $P=.56$ ) survival rates than patients who we excluded, suggesting that selection bias had a minimal impact on our results.

A recursive partitioning survival tree analysis identified the following LN groups with significantly differently survival rates: 0,1 to 11,12 to 29 , and 30 or more LNs examined. These groups were used in our survival analysis. There were significant differences between LN groups with regards to number of potentially important prognostic factors: age, race, geographic location (SEER registry), tumor grade, tumor location, tumor histology, T stage, and timing of radiation therapy (Table 2). We adjusted for these variables in our multivariate survival analysis.

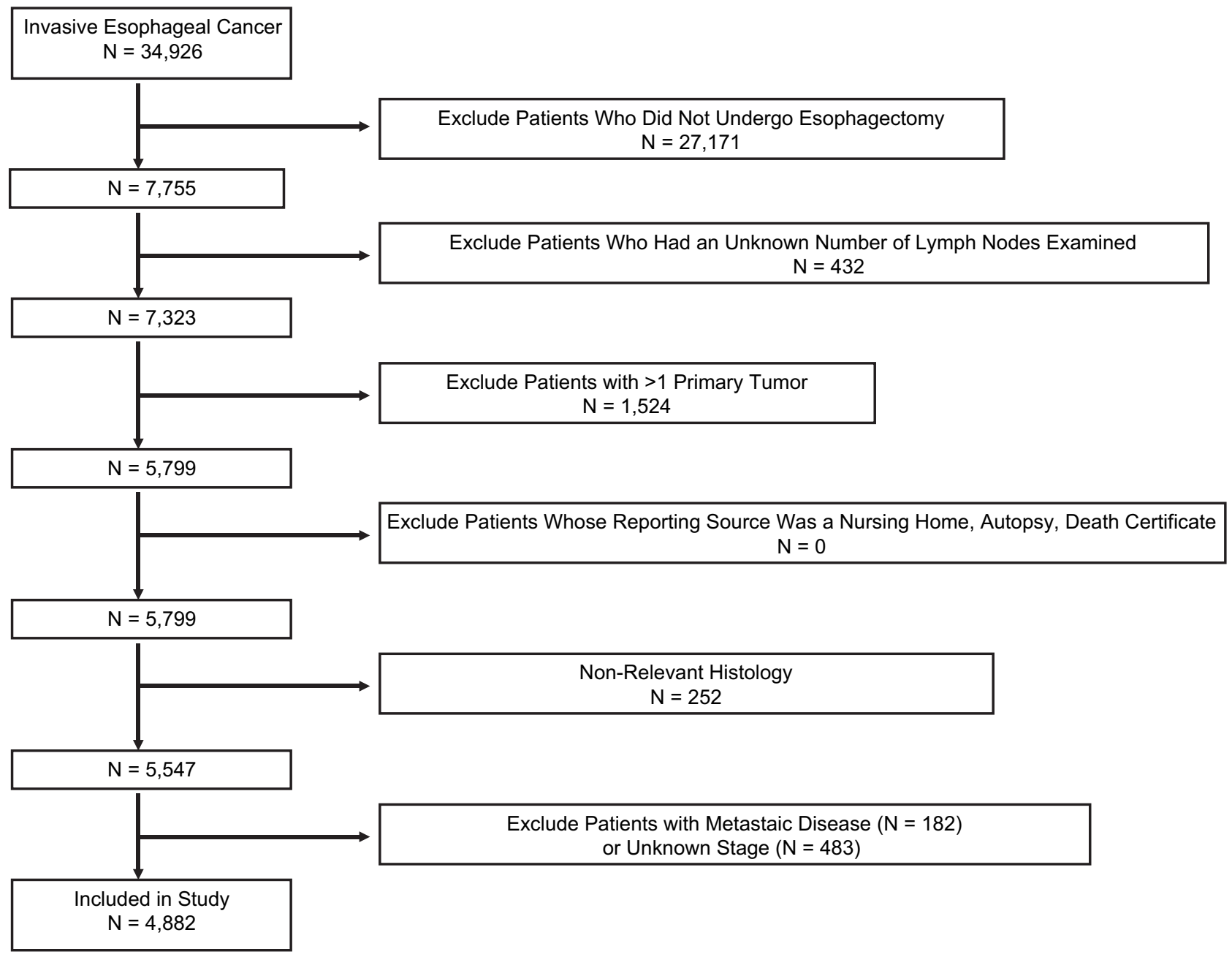

FIGURE 1. Patients included in and excluded from study. 
TABLE 1. Characteristics of patients included versus patients excluded from inclusion in our study*

\begin{tabular}{|c|c|c|c|}
\hline Variable & Patients included & Patients excluded & $P$ value \\
\hline Number of patients & 4882 & 2667 & \\
\hline Age at diagnosis (y) & $62.5 \pm 10.3$ & $64.7 \pm 10.4$ & $<.0001$ \\
\hline Race $(\%)$ & & & .42 \\
\hline Caucasian & 87.0 & 86.8 & \\
\hline African-American & 7.9 & 8.5 & \\
\hline Other & 5.1 & 4.7 & \\
\hline Gender $(\%)$ & & & .35 \\
\hline Men & 81.1 & 80.2 & \\
\hline Women & 18.9 & 19.8 & \\
\hline Tumor location (\%) & & & $<.0001$ \\
\hline Proximal & 3.5 & 6.3 & \\
\hline Midthoracic & 18.4 & 18.3 & \\
\hline Distal & 71.0 & 66.1 & \\
\hline Overlapping & 3.4 & 3.3 & \\
\hline Unknown & 3.7 & 6.0 & \\
\hline Histology $(\%)$ & & & $<.0001$ \\
\hline Adenocarcinoma & 67.9 & 52.7 & \\
\hline $\begin{array}{l}\text { Squamous cell } \\
\text { carcinoma }\end{array}$ & 32.1 & 33.1 & \\
\hline Other & 0 & 14.2 & \\
\hline Grade $(\%)$ & & & $<.0001$ \\
\hline Well differentiated & 6.5 & 4.9 & \\
\hline $\begin{array}{l}\text { Moderately } \\
\text { differentiated }\end{array}$ & 37.2 & 30.9 & \\
\hline $\begin{array}{l}\text { Poorly } \\
\quad \text { differentiated }\end{array}$ & 45.4 & 40.5 & \\
\hline Undifferentiated & 2.0 & 3.8 & \\
\hline Unknown & 8.9 & 19.9 & \\
\hline T stage $(\%)$ & & & $<.0001$ \\
\hline $\mathrm{T} 1$ & 21.1 & 18.5 & \\
\hline $\mathrm{T} 2$ & 16.8 & 16.0 & \\
\hline $\mathrm{T} 3$ & 36.8 & 30.5 & \\
\hline $\mathrm{T} 4$ & 12.1 & 11.1 & \\
\hline Unknown & 13.2 & 23.9 & \\
\hline $\begin{array}{l}\text { Median number } \\
\text { of nodes examined }\end{array}$ & 7 (range, $0-90$ ) & 5 (range, $0-90$ ) & $<.0001$ \\
\hline $\begin{array}{l}\text { Median number } \\
\text { of positive } \\
\text { nodes retrieved }\end{array}$ & 0 (range, $0-35$ ) & 0 (range, $0-46$ ) & .56 \\
\hline $\begin{array}{c}\text { Timing of radiation } \\
\text { therapy }(\%)\end{array}$ & & & $<.0001$ \\
\hline None & 53.4 & 61.1 & \\
\hline Preoperative & 30.4 & 24.8 & \\
\hline Postoperative & 16.2 & 14.1 & \\
\hline
\end{tabular}

\section{Kaplan-Meier Survival Analysis}

We found a significant difference between the LN groups with regards to all-cause (Wilcoxon test, $P<.0001$; Figure $2, A$ ) and cancer-specific mortality (Wilcoxon test, $P=.004 ;$ Figure $2, B)$.

\section{Cox Proportional Hazards Regression Model}

After generating log-log survival plots (not shown) for allcause and cancer-specific mortality, we noted the plots to be
TABLE 2. Characteristics of patients included in our study

\begin{tabular}{|c|c|c|c|c|c|}
\hline & \multicolumn{4}{|c|}{ Number of lymph nodes examined } & \multirow[b]{2}{*}{$P$ value* } \\
\hline & $\mathbf{0}$ & 1 to 11 & 12 to 29 & $\geq \mathbf{3 0}$ & \\
\hline Number of patients & 906 & 2555 & 1263 & 158 & \\
\hline Age at diagnosis $(y), \%$ & & & & & .0019 \\
\hline$<50$ & 21.1 & 47.3 & 28.2 & 3.4 & \\
\hline $50-64$ & 19.4 & 50.1 & 26.9 & 3.6 & \\
\hline$\geq 65$ & 17.1 & 55.7 & 24.4 & 2.8 & \\
\hline Race $(\%)$ & & & & & .0002 \\
\hline Caucasian & 17.8 & 52.7 & 26.3 & 3.2 & \\
\hline African-American & 26.9 & 51.7 & 18.5 & 2.9 & \\
\hline Other & 19.2 & 47.6 & 29.2 & 4.0 & \\
\hline Gender $(\%)$ & & & & & .21 \\
\hline Men & 18.3 & 52.4 & 26.3 & 3.0 & \\
\hline Women & 19.8 & 52.1 & 24.1 & 4.0 & \\
\hline $\begin{array}{l}\text { Tumor location in } \\
\text { esophagus }(\%)\end{array}$ & & & & & $<.0001$ \\
\hline Proximal & 28.9 & 43.9 & 20.2 & 6.4 & \\
\hline Midthoracic & 22.9 & 51.8 & 22.5 & 2.8 & \\
\hline Distal & 16.3 & 53.0 & 27.6 & 3.1 & \\
\hline Overlapping & 26.7 & 46.0 & 20.0 & 7.3 & \\
\hline Unknown & 22.6 & 56.4 & 19.9 & 1.1 & \\
\hline Histology $(\%)$ & & & & & $<.0001$ \\
\hline Adenocarcinoma & 16.3 & 52.7 & 27.7 & 3.3 & \\
\hline $\begin{array}{l}\text { Squamous cell } \\
\text { carcinoma }\end{array}$ & 23.3 & 51.6 & 22.0 & 3.1 & \\
\hline Grade $(\%)$ & & & & & $<.0001$ \\
\hline Well differentiated & 19.4 & 53.4 & 23.4 & 3.8 & \\
\hline $\begin{array}{l}\text { Moderately } \\
\text { differentiated }\end{array}$ & 17.4 & 54.0 & 25.4 & 3.2 & \\
\hline Poorly differentiated & 17.5 & 51.9 & 27.1 & 3.5 & \\
\hline Undifferentiated & 12.5 & 44.8 & 37.5 & 5.2 & \\
\hline Unknown & 29.6 & 48.4 & 20.9 & 1.2 & \\
\hline T stage $(\%)$ & & & & & $<.0001$ \\
\hline $\mathrm{T} 1$ & 17.2 & 56.3 & 23.9 & 2.6 & \\
\hline $\mathrm{T} 2$ & 14.9 & 56.0 & 26.2 & 2.9 & \\
\hline $\mathrm{T} 3$ & 10.6 & 54.9 & 30.9 & 3.6 & \\
\hline $\mathrm{T} 4$ & 19.8 & 48.7 & 27.1 & 4.4 & \\
\hline Unknown & 46.7 & 37.5 & 13.5 & 2.3 & \\
\hline $\begin{array}{c}\text { Timing of radiation } \\
\text { therapy }(\%)\end{array}$ & & & & & $<.0001$ \\
\hline None & 13.6 & 55.0 & 27.6 & 3.8 & \\
\hline Preoperative & 29.4 & 48.4 & 20.1 & 2.1 & \\
\hline Postoperative & 14.7 & 51.7 & 29.9 & 3.7 & \\
\hline
\end{tabular}

relatively homogenous over time; there was only a minor deviation at later periods of follow-up. Therefore, we chose to exclude the time-dependent interaction term from our final model because including the term would have significantly increased the complexity of our analysis, with little improvement in accuracy.

When modeled as a continuous variable, we noted a significant relationship between all-cause $(P<.0001)$ and cancer-specific mortality $(P=.0002)$ : survival improved as the number of LNs examined increased. To determine whether 


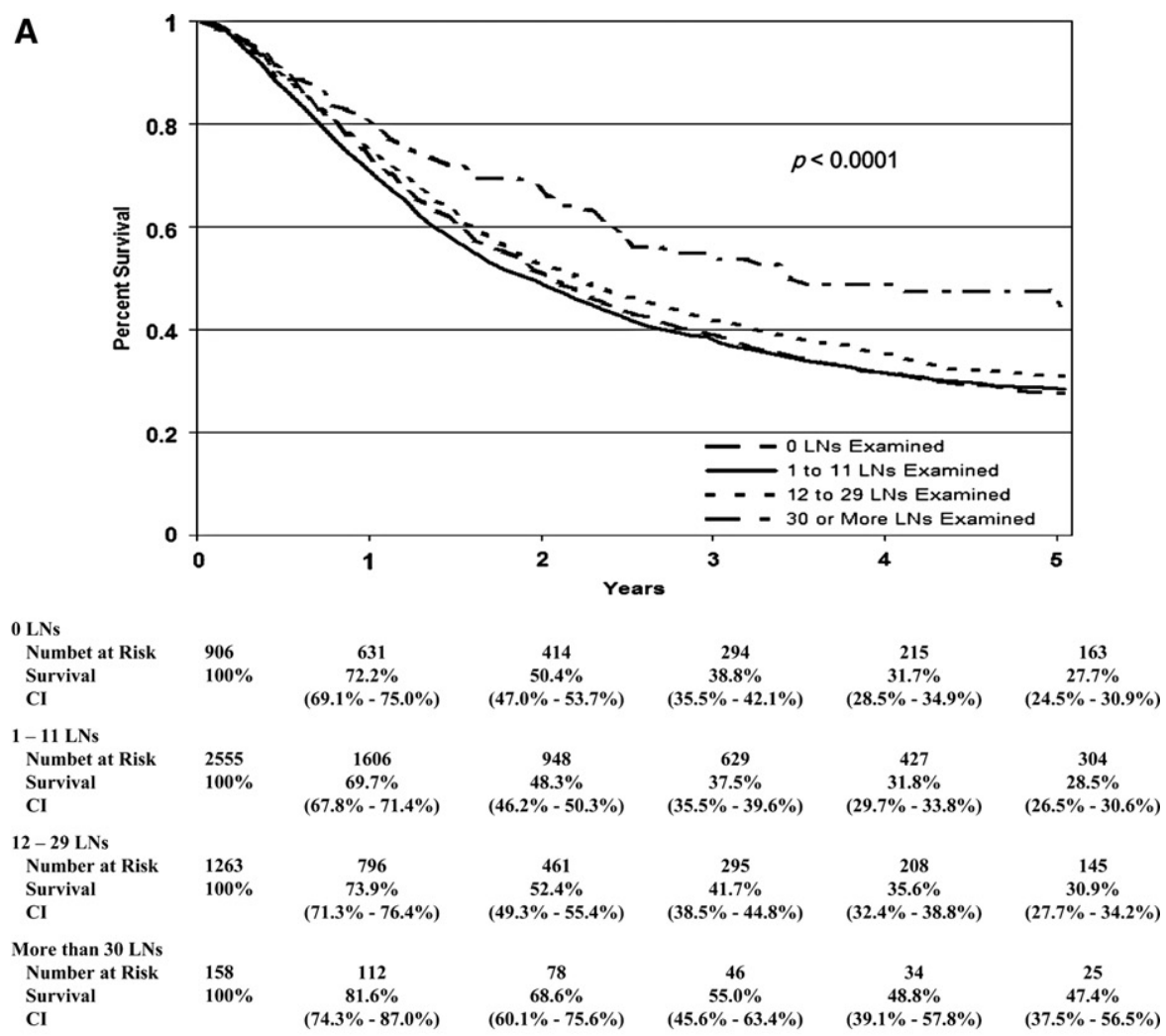

B

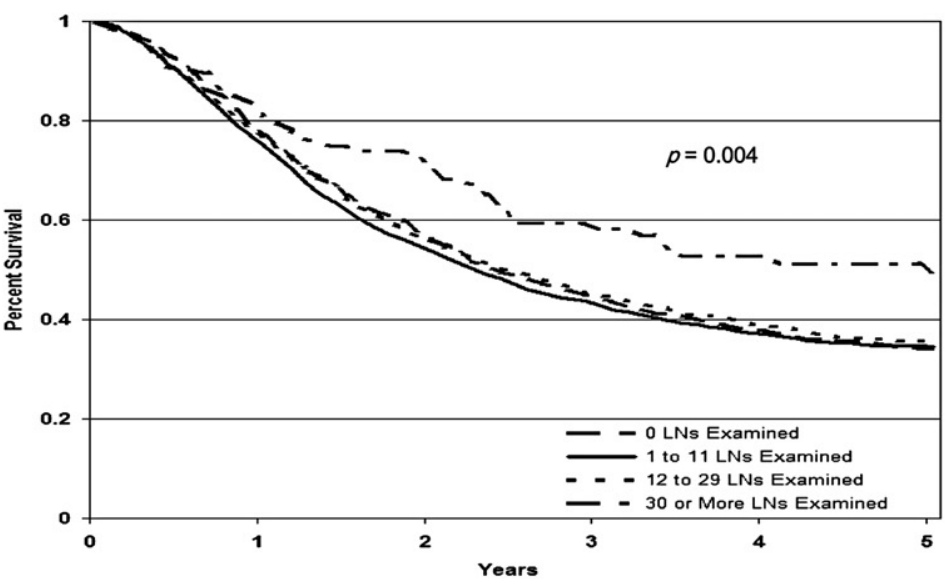

$0 \mathrm{Ls}$

Numbet

Survival

898
$100 \%$

625
$77.1 \%$
$(74.1 \%-79.7 \%$

45.6\% - $63.4 \%$

$(39.1 \%-57.8 \%) \quad(37.5 \%-56.5 \%)$

1 - 11 LNs

Numbet at
Survival

CI

$2538-1599$

293

293

$100 \%$

$\mathbf{7 4 . 8} \%$

(73.0\% - 76.5\%)

$44.8 \%$
$(41.2 \%-48.3 \%)$

$38.1 \%$

$12-29$ LNs

Number at Risk

Survival

CI

1254
$100 \%$

793

$\begin{array}{cc}\mathbf{7 6 3} & \mathbf{4 5 9} \\ \mathbf{( 7 3 . 9} \%-\mathbf{7 8 . 9} \%) & \mathbf{5 5 . 7 \%} \\ & \end{array}$

$\mathbf{9 4 2}$
$\mathbf{5 3 . 7} \%$

$51.5 \%-55.8 \%)$

626
$43.0 \%$

26
$-45.2 \%)$

425
$37.1 \%$
$(34.8 \%-39.4 \%)$

More than $30 \mathrm{LNs}$

Number at Risk

Survival
CI

153

$100 \%$

293
$45.1 \%$

$38.8 \%$

(35.4\% - 42.2\%)

304
$34.7 \%$

(32.3\% - 37.0\%)

108
$83.5 \%$
$(76.2 \%-88.7 \%)$

${ }^{77}$

$\begin{array}{cc}77 & 46 \\ 73.0 \% & 59.5 \% \\ (64.6 \%-79.8 \%) & (49.8 \%-67.9\end{array}$

$\begin{array}{cc}\mathbf{4 6} & \mathbf{3 4} \\ \mathbf{5 9 . 5} \% & \mathbf{5 2 . 8} \%\end{array}$

(42.6\% - 62.0\%)

145
$35.8 \%$

(32.3\% - 39.2\%)

FIGURE 2. Overall (A) and cancer-specific (B) survival Kaplan-Meier curves for each lymph node $(L N)$ group. Survival with $95 \%$ confidence interval $(C I)$ at each year of follow-up for each LN group provided in the table below each graph. 
TABLE 3. Cox proportional hazards regression models for all-cause and cancer-specific mortality

\begin{tabular}{|c|c|c|}
\hline Variable & $\begin{array}{c}\text { All-cause mortality } \\
\text { HR }(95 \% \text { CI })^{*}, \dagger\end{array}$ & $\begin{array}{c}\text { Cancer-specific } \\
\text { mortality HR } \\
(95 \% \text { CI })^{*}, \dagger\end{array}$ \\
\hline \multicolumn{3}{|l|}{$\begin{array}{l}\text { Number of lymph } \\
\text { nodes examined* }\end{array}$} \\
\hline 0 & 1.00 (reference) $^{\mathrm{a}}$ & 1.00 (reference) $^{\mathrm{a}}$ \\
\hline $1-11$ & $0.98(0.88,1.08)^{\mathrm{a}}$ & $0.99(0.88,1.11)^{\mathrm{a}}$ \\
\hline $12-29$ & $0.85(0.75,0.95)^{\mathrm{b}}$ & $0.90(0.79,1.02)^{\mathrm{a}}$ \\
\hline$\geq 30$ & $0.55(0.42,0.72)^{\mathrm{c}}$ & $0.58(0.44,0.78)^{\mathrm{b}}$ \\
\hline \multicolumn{3}{|l|}{ Age at diagnosis (y) } \\
\hline$<50$ & 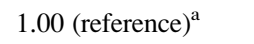 & 1.00 (reference) $^{\mathrm{a}}$ \\
\hline $50-64$ & $1.14(0.99,1.31)^{\mathrm{a}}$ & $1.08(0.94,1.25)^{\mathrm{a}}$ \\
\hline$\geq 65$ & $1.51(1.32,1.73)^{\mathrm{b}}$ & $1.37(1.19,1.58)^{\mathrm{b}}$ \\
\hline \multicolumn{3}{|l|}{ Race } \\
\hline Caucasian & 1.00 (reference) $^{\mathrm{a}}$ & $1.00{\text { (reference })^{\mathrm{a}}}$ \\
\hline African-American & $1.24(1.08,1.44)^{\mathrm{b}}$ & $1.23(1.05,1.44)^{\mathrm{a}}$ \\
\hline Other & $0.93(0.76,1.12)^{\mathrm{a}}$ & $1.00(0.82,1.23)^{\mathrm{a}}$ \\
\hline \multicolumn{3}{|l|}{ Gender } \\
\hline Female & 1.00 (reference) $^{\mathrm{a}}$ & 1.00 (reference) $^{\mathrm{a}}$ \\
\hline Male & $1.21(1.10,1.35)^{\mathrm{b}}$ & $1.21(1.09,1.35)^{\mathrm{b}}$ \\
\hline \multicolumn{3}{|l|}{$\begin{array}{c}\text { Tumor location in } \\
\text { esophagus }\end{array}$} \\
\hline Proximal & 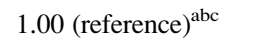 & 1.00 (reference) $^{\mathrm{a}}$ \\
\hline Midthoracic & $1.23(0.99,1.51)^{\mathrm{ac}}$ & $1.29(1.02,1.62)^{\mathrm{b}}$ \\
\hline Distal & $0.99(0.81,1.22)^{\mathrm{bc}}$ & $1.01(0.81,1.27)^{\mathrm{a}}$ \\
\hline Overlapping & $1.20(0.91,1.58)^{\mathrm{c}}$ & $1.21(0.90,1.64)^{\mathrm{a}}$ \\
\hline Unknown & $1.19(0.89,1.58)^{\mathrm{c}}$ & $1.16(0.84,1.60)^{\mathrm{a}}$ \\
\hline \multicolumn{3}{|l|}{ Histology } \\
\hline Adenocarcinoma & 1.00 (reference) $^{\mathrm{a}}$ & 1.00 (reference) $^{\mathrm{a}}$ \\
\hline $\begin{array}{l}\text { Squamous cell } \\
\text { carcinoma }\end{array}$ & $1.13(1.02,1.25)^{\mathrm{b}}$ & $1.12(1.01,1.25)^{\mathrm{b}}$ \\
\hline \multicolumn{3}{|l|}{ Grade } \\
\hline Well differentiated & 1.00 (reference) $^{\mathrm{a}}$ & $1.00{\text { (reference })^{\mathrm{a}}}$ \\
\hline $\begin{array}{l}\text { Moderately } \\
\text { differentiated }\end{array}$ & $1.58(1.30,1.91)^{\mathrm{b}}$ & $1.82(1.45,2.29)^{\mathrm{b}}$ \\
\hline Poorly differentiated & $1.99(1.65,2.41)^{\mathrm{c}}$ & $2.40(1.92,3.01)^{\mathrm{c}}$ \\
\hline Undifferentiated & $2.04(1.51,2.77)^{\mathrm{c}}$ & $2.41(1.71,3.39)^{\mathrm{c}}$ \\
\hline Unknown & $1.28(1.02,1.62)^{\mathrm{d}}$ & $1.49(1.14,1.96)^{\mathrm{d}}$ \\
\hline \multicolumn{3}{|l|}{ T stage } \\
\hline $\mathrm{T} 1$ & 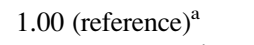 & 1.00 (reference) $^{\mathrm{a}}$ \\
\hline $\mathrm{T} 2$ & $2.16(1.87,2.49)^{\mathrm{b}}$ & $2.52(2.14,2.96)^{b}$ \\
\hline $\mathrm{T} 3$ & $2.93(2.58,3.34)^{\mathrm{c}}$ & $3.43(2.96,3.98)^{\mathrm{c}}$ \\
\hline $\mathrm{T} 4$ & $3.78(3.26,4.39)^{\mathrm{d}}$ & $4.60(3.90,5.43)^{\mathrm{d}}$ \\
\hline Unknown & $2.22(1.90,2.58)^{\mathrm{b}}$ & $2.47(2.08,2.95)^{\mathrm{b}}$ \\
\hline \multicolumn{3}{|l|}{$\begin{array}{l}\text { Timing of radiation } \\
\text { therapy }\end{array}$} \\
\hline None & 1.00 (reference) $^{\mathrm{a}}$ & 1.00 (reference) $^{\mathrm{a}}$ \\
\hline Preoperative & $0.74(0.67,0.81)^{\mathrm{b}}$ & $0.75(0.68,0.83)^{\mathrm{b}}$ \\
\hline Postoperative & $0.90(0.81,0.99)^{\mathrm{c}}$ & $0.95(0.85,1.06)^{\mathrm{a}}$ \\
\hline
\end{tabular}

or not there was a threshold (above which there is no significant improvement in morality), we also modeled the association between the LN groups and mortality. Only patients who had at least 12 LNs evaluated had significantly lower all-cause and cancer-specific mortality rates than patients who had no LNs examined. Patients with more than 30 LNs had the lowest mortality rates (Table 3 ). Even after excluding patients who died within 30 days of esophagectomy, our results were unchanged.

Our multivariate assessment also revealed that patients who were 65 and older, men, or African-Americans and patients with squamous cell carcinomas and higher-grade and more advanced T-stage tumors had worse all-cause and cancer-specific mortality (Table 3). Patients with midthoracic tumors (where squamous cell carcinomas predominate) had worse cancer-specific mortality and a nonsignificant trend toward worse all-cause mortality as compared with other tumor locations. Neoadjuvant radiation therapy had a protective effect. Compared with patients who did not undergo radiation therapy, patients who had adjuvant radiation therapy had slightly lower all-cause mortality rates; however, cancer-specific mortality was not improved (Table 3 ).

Due to concern for multicollinearity, we did not include the number of positive LNs in our multivariate survival model. Instead, we stratified our results by LN positivity to adjust for the confounding influence of LN status on survival and found that our results were unchanged (data not shown).

We assessed for interactions between $\mathrm{LN}$ counts and other tumor characteristics. The test for heterogeneity across T-stage strata was significant $(P<.0001)$. However, after stratifying our analysis by $\mathrm{T}$ stage (to ensure that our pooled estimates were consistent across these strata), our results were unchanged. We did not find significant heterogeneity across histology strata $(P=.16)$, and our results were unchanged when we stratified by histology. We found significant heterogeneity across tumor grade strata $(P=.002)$. Our results were unchanged when we stratified our analysis for moderately differentiated and poorly differentiated tumors. We were unable to stratify by all tumor grades as few patients with more than 30 LNs examined had well-differentiated $(n=12)$ or undifferentiated tumors $(\mathrm{n}=7)$.

Our results confirmed our hypothesis that patients who had neoadjuvant radiation therapy had significantly lower $(P<.0001)$ LN yields (median, 5; range, $0-71)$ than patients who did not (median, 7; range 0-90). Though the test for heterogeneity across timing of radiation strata was significant $(P<.0001)$, stratifying our results by timing of radiation therapy did not change our study findings: (1) there is an association between higher LN counts and reduced mortality and (2) patients with a least $30 \mathrm{LNs}$ examined had the lowest mortality rates.

\section{LN Counts and Nodal Staging}

We plotted the probability of finding at least 1 positive $\mathrm{LN}$ by the number of LNs examined using locally weighted least 


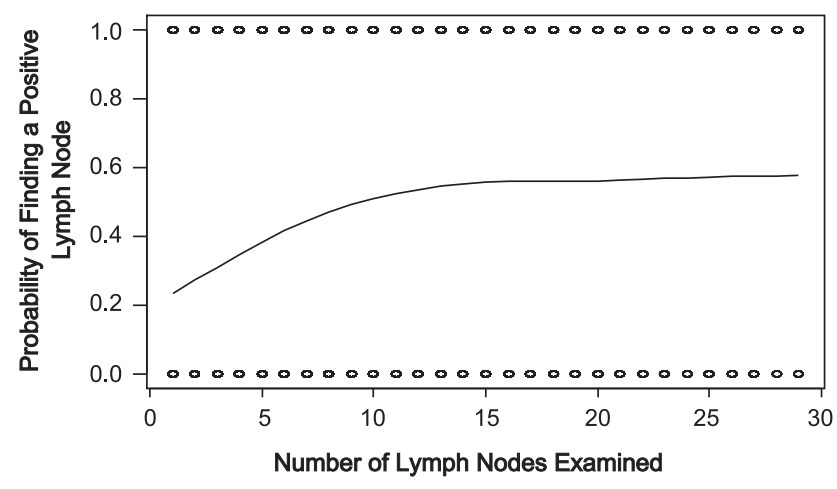

FIGURE 3. Locally weighted least squares smooth of the percentage of patients with at least 1one positive lymph node as function of the number of lymph nodes examined.

squares smoothing (Figure 3). The slope of the curve was largest (indicative of the greatest impact on finding LN metastasis) when at least $3 \mathrm{LNs}$ were examined, as compared with examination of less than 3 LNs (adjusted odds ratio [OR] 2.97; 95\% confidence interval [CI]: 2.32-3.79). The probability of finding a positive LN reaches an asymptote near 15 LNs examined, which suggests that higher LN yields do not improve the accuracy of nodal staging (Figure 3). Indeed, patients who had 12 to 29 LNs examined were significantly more likely to have LN metastasis detected than patients with 11 or fewer LNs examined (OR 1.69; 95\% CI: 1.44-1.98). There was no significant difference in the odds of detecting LN metastasis between patients who had 30 or more LNs examined and patients who had 12 to 29 LNs examined (OR 0.93, 95\% CI: 0.63-1.37).

\section{DISCUSSION}

Using a large US population-based cancer registry, we demonstrated that the number of pathologically examined LNs removed at the time of esophagectomy has a significant association with all-cause and cancer-specific mortality. We were not able to ascertain a threshold of mortality benefit; survival was maximized when more than $30 \mathrm{LNs}$ are examined, independent of patient characteristics, tumor characteristics, and use of neoadjuvant radiation therapy. Though the inclusion criteria and methodologies are different, our results corroborate other recently published single-institution, international, and population-based studies that have examined the association between LN counts and survival in esophageal cancer patients. ${ }^{5-9}$

Bollschweiler and colleagues ${ }^{6}$ performed a single-institution retrospective review of 213 esophageal cancer patients who had surgical resection with curative intent (predominately en bloc esophagectomy and 2-field lymphadenectomy) and determined that at least 16 LNs should be examined. As compared with patients who had 16 to 30 LNs examined, they did not find an improvement in survival if more than 30 LNs were examined; however, only 7 patients with more than $30 \mathrm{LNs}$ examined had 4 or more years of follow-up.

Altorki and colleagues ${ }^{5}$ reviewed their experience of 264 patients who underwent esophagectomy without neoadjuvant therapy. ${ }^{5}$ Patients were divided into quartiles based on the number of LNs examined. They found that higher LN counts were associated with improved survival. Similar to our results, they found that patients with more than 26 LNs examined had the most favorable survival rates. Unlike our results, however, their results differed based on LN status. In patients with N0 disease, survival was maximized when 26 LNs were examined. Patients with N1 disease, however, required examination of more than $40 \mathrm{LNs}$ to achieve a significant improvement in survival as compared with patients who had 16 or fewer LNs examined. After stratifying by LN positivity, we did not find a difference in the number of LNs that should be examined; survival was maximized when more than 30 nodes were examined, regardless of LN status. Differences in our patient population, sample size, inclusion criteria (we did not exclude patients underwent neoadjuvant radiation therapy), and variables included in our multivariate survival model may explain this discrepancy between our results and the results of others.

The results of a retrospective, international, multi-institutional study of 2303 patients who underwent $\mathrm{R} 0$ resection of esophageal cancer were recently published by Peyre and associates. ${ }^{8}$ Similar to other studies, they excluded patients who had neoadjuvant or adjuvant therapy. They dichotomized patients into LN groups using thresholds of 1 to 60 LNs examined (ie, $\leq 1$ vs $>1, \leq 2$ vs $>2$, etc) and found that the chi-square for the $\mathrm{LN}$ variable in a Cox proportional hazards regression model was maximized (suggestive of the greatest effect) between 23 and 29 LNs examined. Similar to our results, however, they did not identify a threshold LN count above which there was no incremental improvement in survival; survival continued to improve as more LNs were examined.

Recently, Greenstein and colleagues ${ }^{7}$ utilized the SEER database (1988-2003) to assess the association between negative LN counts and survival in patients with node-negative (AJCC stage I and IIa) esophageal carcinoma who did not have neoadjuvant radiation therapy. Unlike our study, they found heterogeneity across $\mathrm{T}$-stage strata. For patients with $\mathrm{T} 1$ disease, they determined that higher LN yields (more than $18 \mathrm{LNs}$ ) were necessary to maximize survival. However, for patients with T2 or T3 disease, examination of 11 to 17 LNs provided the same survival advantage as examination of more than 18 LNs. Differences in methodology may explain this discrepancy. Greenstein and colleagues ${ }^{7}$ focused on number of negative LNs examined; we focused on total $\mathrm{LN}$ counts. They restricted their analysis to patients with localized disease; we included patients with localized and regional disease. They excluded patients who had 
neoadjuvant therapy; we did not. In addition to adenocarcinomas and squamous cell carcinomas, they also included other histologies (small cell carcinomas, lymphomas, melanomas, and sarcomas). We chose to exclude these tumors as they likely have different biologic behavior than adenocarcinomas and squamous cell carcinomas of the esophagus.

In contrast to many of these previous studies, ${ }^{5,7,8}$ we chose to include patients who had neoadjuvant radiation therapy because it is becoming the standard of care for locally advanced esophageal carcinoma. ${ }^{10}$ Therefore, our results may be more generalizable to today's esophageal cancer patient. We found that the esophageal specimens from patients who complete neoadjuvant radiation therapy have significantly fewer LNs than patients who did not, which is not surprising as radiation therapy may cause lymphocyte depletion and stromal fibrosis resulting in significantly smaller (or even ablated) LNs. ${ }^{11}$ Nonetheless, LN counts are still important in patients who have preoperative radiation therapy; radiation therapy should not be an excuse for not finding LNs in an esophagectomy specimen.

Though our results indicate that a large number of LNs should be examined to maximize all-cause and cancerspecific mortality, it does not provide insight into the debates regarding the optimal esophagectomy approach (ie, transhiatal, Ivor Lewis, and en bloc) or the optimal extent of lymphadenectomy (2-field vs 3-field). Our results simply indicate that 30 or more LNs should be examined (regardless of the operative technique).

Though it is clear that LN counts are associated with survival, the underlying mechanisms behind this association are poorly understood. There are a number of possibilities. One such mechanism is improved local tumor control: clearing more LNs (to ensure removal of occult or overt metastasis) may reduce local recurrence rates. Indeed, recurrent disease is almost uniformly fatal. ${ }^{12}$ Unfortunately, SEER does not collect recurrence data, preventing us from further analyzing this potential association.

More accurate cancer staging is another potential mechanism. By removing more LNs, the risk of failing to detect LN metastasis (which may prevent patients from receiving optimal cancer therapy) is lessened. However, we demonstrated that the number of LNs needed to optimize cancer-specific mortality ( 30 or more LNs) is not the same as the number need to maximize the likelihood of detecting $\mathrm{LN}$ metastasis (about $15 \mathrm{LNs}$ ). Our findings are similar to a recursive partitioning analysis of data from 336 patients who had esophagectomy at the Memorial Sloan-Kettering Cancer Center; examination of $18 \mathrm{LNs}$ was recommended to optimize the accuracy of cancer staging. ${ }^{13}$ This discordance between the number of LNs that should be examined to optimize survival and the number needed to optimize cancer staging has been replicated in the colorectal cancer literature. According to current guidelines, at least $12 \mathrm{LNs}$ should be examined in colorectal cancer patients who undergo colectomy. ${ }^{14}$ In a study using the SEER-Medicare database, Wong et al ${ }^{15}$ demonstrated that removing $12 \mathrm{LNs}$ did not increase the accuracy of colorectal cancer staging.

The discordance between the number of LNs needed to optimize survival and the number needed to accurately stage patients suggests that potential factors are present in both esophageal and colorectal cancer patients (and likely patients with other malignancies as well) that confound the association between LN counts and survival. Such factors include: (1) surgeon factors (training, esophagectomy volume, and oncologic quality of the esophagectomy and lymphadenectomy); (2) pathologist factors (skill and diligence looking for LNs); (3) hospital factors (esophagectomy volume, teaching status, and global quality of patient care); (4) patient factors (ability to mount an immune response to cancer thereby making it easier to find LNs); or (5) other (unrecognized) factors.

There are several limitations of our study, some of which are inherent limitations of SEER. In particular, the SEER cancer registries do not collect information on several factors associated with survival, such as performance status, ${ }^{16}$ comorbidities,${ }^{17}$ use of chemotherapy, ${ }^{18}$ completeness of resection, ${ }^{19}$ margin length,${ }^{20}$ time to local recurrence, ${ }^{19}$ weight loss (more than $10 \%$ ), ${ }^{21}$ and surgeon and hospital esophagectomy volume. ${ }^{22,23}$ Therefore, we could not adjust for these covariates in our model. In addition, SEER does not mandate endoscopic verification of tumor location. As a result, there is a potential for misclassification. However, when we stratified patients into broader tumor location groups (cervical, thoracoabdominal, and overlapping), our results were unchanged, indicating that misclassification of tumor location was not a significant source of error.

Finally, we restricted our analysis to only include adenocarcinomas and squamous cell carcinomas to reduce the confounding influence of other histologies (and their varying biologic behavior) on our results. As a result, our results may not be generalizable to other histologies.

We believe our use of a large population-based cancer registry, inclusion of patients representative to today's esophageal cancer patients (ie, those who had neoadjuvant therapy), and demonstration of consistent results across a variety of strata adds to the growing body of evidence in the literature regarding the association between $\mathrm{LN}$ counts and survival. Additional studies are needed to ascertain the underlying mechanisms behind LN counts and survival.

\section{CONCLUSION}

Though the underlying mechanism has yet to be elucidated, the number of LNs examined in esophageal cancer patients who undergo esophagectomy is a significant independent determinant of all-cause and cancer-specific mortality, even among patients who complete neoadjuvant radiation therapy. Therefore, LN counts after esophageal 
resection should be a central feature of surgical quality assessment.

\section{References}

1. Surveillance, Epidemiology, and End Results (SEER) Program. Limited-Use Data (1973-2005), National Cancer Institute, DCCPS, Surveillance Research Program, Cancer Statistics Branch. Released April 2008, based on the November 2007 submission. Available at: www.seer.cancer.gov.

2. Fritz AG, Percy C, Jack A, Sobin LH. International Classification of Diseases for Oncology. 3rd ed. Geneva: World Health Organization; 2000.

3. Zhang HP, Singer B. Recursive Partitioning in the Health Sciences. New York: Springer; 1999.

4. Greene FL, Page DL, Fleming ID, Fritz AG. AJCC Cancer Staging Manual. 6th ed. New York: Springer-Verlag; 2002.

5. Altorki NK, Zhou XK, Stiles B, Port JL, Paul S, Lee PC, et al. Total number of resected lymph nodes predicts survival in esophageal cancer. Ann Surg. 2008; 248:221-6.

6. Bollschweiler E, Baldus SE, Schroder W, Schneider PM, Holscher AH. Staging of esophageal carcinoma: length of tumor and number of involved regional lymph nodes. Are these independent prognostic factors? J Surg Oncol. 2006;94:355-63.

7. Greenstein AJ, Litle VR, Swanson SJ, Divino CM, Packer S, Wisnivesky JP. Effect of the number of lymph nodes sampled on postoperative survival of lymph node-negative esophageal cancer. Cancer. 2008;112:1239-46.

8. Peyre CG, Hagen JA, DeMeester SR, Altorki NK, Ancona E, Griffin SM, et al. The number of lymph nodes removed predicts survival in esophageal cancer: an international study on the impact of extent of surgical resection. Ann Surg. 2008;248:549-56.

9. Schwarz RE, Smith DD. Clinical impact of lymphadenectomy extent in resectable esophageal cancer. J Gastrointest Surg. 2007;11:1384-93; discussion 93-4.

10. The National Comprehensive Cancer Network. NCCN Clinical Practice Guidelines in Oncology: Esophageal Cancer, VI 2008. Available at: http://www. nccn.org/professionals/physician_gls/PDF/esophageal.pdf. Updated March 12, 2008. Accessed May 1, 2008.

11. Habr-Gama A, Perez RO, Proscurshim I, Rawet V, Pereira DD, Sousa AH, et al. Absence of lymph nodes in the resected specimen after radical surgery for distal rectal cancer and neoadjuvant chemoradiation therapy: what does it mean? Dis Colon Rectum. 2008;51:277-83.
12. Altorki N, Kent M, Ferrara C, Port J. Three-field lymph node dissection for squamous cell and adenocarcinoma of the esophagus. Ann Surg. 2002;236: 177-83.

13. Rizk N, Venkatraman E, Park B, Flores R, Bains MS, Rusch V. The prognostic importance of the number of involved lymph nodes in esophageal cancer: implications for revisions of the American Joint Committee on Cancer staging system. J Thorac Cardiovasc Surg. 2006;132:1374-81.

14. Cserni G, Vinh-Hung V, Burzykowski T. Is there a minimum number of lymph nodes that should be histologically assessed for a reliable nodal staging of T3N0M0 colorectal carcinomas? J Surg Oncol. 2002;81:63-9.

15. Wong SL, Ji H, Hollenbeck BK, Morris AM, Baser O, Birkmeyer JD. Hospital lymph node examination rates and survival after resection for colon cancer. JAMA. 2007;298:2149-54.

16. Tanisada K, Teshima T, Ikeda H, Abe M, Owen JB, Hanks GE, et al. A preliminary outcome analysis of the Patterns of Care Study in Japan for esophageal cancer patients with special reference to age: non surgery group. Int J Radiat Oncol Biol Phys. 2000;46:1223-33.

17. Steyerberg EW, Neville B, Weeks JC, Earle CC. Referral patterns, treatment choices, and outcomes in locoregional esophageal cancer: a population-based analysis of elderly patients. J Clin Oncol. 2007;25:2389-96.

18. Thirion PG, Michiels S, Le Maître A, Tierney J, on behalf of the MetaAnalysis of Chemotherapy in Esophagus Cancer Collaborative Group. Individual patient data-based meta-analysis assessing preoperative chemotherapy in resectable oesophageal carcinoma. J Clin Oncol. 2007;25(18S):4512.

19. Schipper PH, Cassivi SD, Deschamps C, Rice DC, Nichols FC III, Allen MS, et al. Locally recurrent esophageal carcinoma: when is re-resection indicated? Ann Thorac Surg. 2005;80:1001-5; discussion 5-6.

20. Barbour AP, Rizk NP, Gonen M, Tang L, Bains MS, Rusch VW, et al. Adenocarcinoma of the gastroesophageal junction: influence of esophageal resection margin and operative approach on outcome. Ann Surg. 2007;246:1-8.

21. Mariette C, Taillier G, Van Seuningen I, Triboulet JP. Factors affecting postoperative course and survival after en bloc resection for esophageal carcinoma. Ann Thorac Surg. 2004;78:1177-83.

22. Connors RC, Reuben BC, Neumayer LA, Bull DA. Comparing outcomes after transthoracic and transhiatal esophagectomy: a 5-year prospective cohort of 17,395 patients. J Am Coll Surg. 2007;205:735-40.

23. Birkmeyer JD, Sun Y, Wong SL, Stukel TA. Hospital volume and late survival after cancer surgery. Ann Surg. 2007;245:777-83. 